\title{
The Cedar Project WelTel mHealth intervention for HIV prevention in young Indigenous people who use illicit drugs: study protocol for a randomized controlled trial
}

Kate Jongbloed', Anton J. Friedman², Margo E. Pearce ${ }^{2}$, Mia L. Van Der Kop ${ }^{3,4}$, Vicky Thomas², Lou Demerais ${ }^{5}$, Sherri Pooyak ${ }^{6}$, Martin T. Schechter ${ }^{1}$, Richard T. Lester ${ }^{7}$, Patricia M. Spittal ${ }^{1 *}$ and The Cedar Project Partnership

\begin{abstract}
Background: Despite successes in preventing and treating HIV, Indigenous people in Canada continue to face disproportionately high rates of HIV infection. Programs that support healing from lifetime trauma, support connection to culture, and reduce drug-related harms are critical to preventing HIV among young Indigenous people who use drugs. The Cedar Project WelTel mHealth intervention proposed here is a structured mobile-phone initiative to connect young Indigenous people who use drugs with Cedar Case Managers in a community-based setting. The intervention consists of a package of supports, including a mobile phone and cellular plan, weekly two-way text messaging, and support from Cedar Case Managers.

Methods: The Cedar Project WelTel mHealth study is a multi-site Zelen pre-randomized trial to measure the effect of a two-way supportive text-message intervention to reduce HIV vulnerability among young Indigenous people who use illicit drugs in two Canadian cities. The trial is nested within the Cedar Project, an ongoing cohort study addressing HIV and hepatitis C vulnerability among young Indigenous people who use drugs in Vancouver and Prince George, British Columbia. The Cedar Project Partnership, an independent body of Indigenous Elders, leaders, and health/social service experts, governs all aspects of the study. Two hundred participants will be followed over a 16-month period, with HIV propensity score at 6 months as the primary outcome. Secondary outcomes include HIV propensity at 1 year, HIV risk, resilience, psychological distress, access to drug-related services, and connection to culture measured at 6 months and 1 year. Primary analysis is by intention to treat.
\end{abstract}

Discussion: Culturally safe interventions that address barriers to HIV prevention while supporting the strength of young Indigenous people who use drugs are urgently needed. Despite presenting a tremendous opportunity to connect young, highly transient Indigenous people who use drugs to prevention services, supportive two-way mHealth programs have yet to be tested for HIV prevention in a community-based setting with this population.

Trial registration: ClinicalTrials.gov NCT02437123 https://clinicaltrials.gov/show/NCT02437123 (registered 4 May 2015). Protocol version: 24 July 2015.

Keywords: mHealth, Indigenous, HIV prevention, Substance use

\footnotetext{
* Correspondence: spittal@sm.hivnet.ubc.ca

'School of Population and Public Health, Faculty of Medicine, University of

British Columbia, 2206 East Mall, Vancouver, BC V6T 1Z3, Canada

Full list of author information is available at the end of the article
} 


\section{Background}

Indigenous scholars suggest that understanding substance use and HIV vulnerability among young Indigenous people must begin with consideration of the ongoing impact of colonization and intergenerational trauma while also acknowledging strength and survival in the face of incredible hardships [1-5]. Indigenous scholars have described intergenerational trauma as a collective emotional and psychological "soul wound" that continues to affect the youngest generations of Indigenous people [6, 7]. In Canada, well over 150,000 Indigenous children were forcibly removed from their homes and placed in residential schools between 1883 and 1996 [8]. The persistent devaluation of Indigenous cultural identity and widespread physical, sexual, and emotional abuse that occurred at residential schools has had far-reaching consequences for the health of Indigenous people [9]. Many Indigenous scholars and advocates argue that cultural oppression through the removal of children from their families and communities has continued through the child welfare system [10]. In fact, estimates indicate that three times as many Indigenous children are currently in care of the state compared with during the peak of the residential school system in the 1940s [10]. In a study of young Indigenous people who use drugs in British Columbia (BC), $65 \%$ had been removed from their biological parents and placed into care [11]. Those who had been in care were 2.1 times more likely to have a parent who attended residential school and 2.6 times more likely to have been sexually abused. The residential school and child welfare systems in Canada disrupted ways of life that had sustained Indigenous families and communities over generations, and introduced cycles of multi-generational grief, trauma, and displacement [10]. Acknowledging the profound effect of intergenerational and lifetime trauma, and subsequent reliance on the powerful numbing effect of drugs, is critical to understanding HIV vulnerability among young Indigenous people who use drugs [4, 7, 12-15].

Indigenous leaders in Canada are justifiably concerned about alarming rates of HIV infection among their young people who continue to be vastly over-represented in the HIV epidemic [16]. Although Indigenous people comprise just $3.8 \%$ of Canada's total population, Indigenous people in 2013 represented $16 \%$ of positive HIV tests in the country [17]. Injection drug use remains the primary exposure among Indigenous people, accounting for $64 \%$ of HIV infections, compared with $11 \%$ among nonIndigenous people [16]. In addition, one third of new infections were among young Indigenous people under 30 years of age, compared with only $20 \%$ among young non-Indigenous people [16]. There is a continuing crisis of HIV infection among young Indigenous people who use injection and non-injection drugs in BC; HIV prevalence reached $9 \%$ overall and $17 \%$ among those who inject drugs [12]. In addition, HIV incidence among young Indigenous people who use injection drugs in $\mathrm{BC}$ is estimated to be three times higher than among nonindigenous young people [18].

Significant and multi-layered barriers to HIV prevention services, including health care, harm reduction, addiction treatment, and safer sex work environments, have been identified among young Indigenous people who use drugs $[2,3,12,13]$. Young Indigenous people who use drugs have described frequent encounters with systemic and interpersonal racism, stigma and judgement within harm reduction and health services [1, 19-23]. Furthermore, despite compelling evidence that harm reduction programs, including supervised drug consumption facilities [24] and opioid substitution therapy [25], are effective at preventing HIV infection, studies indicate that young Indigenous people are failing to engage with these services $[12,18,26,27]$. Finally, safety in sex work, as well as supports to leave sex work if desired, remains elusive in a country where young Indigenous women in sex work routinely face extreme violence and death in the course of their work [28, 29].

Addressing barriers to HIV prevention among young Indigenous people who use drugs-while also acknowledging resilience despite adversity-is urgently required. In New Zealand, Maori nurses have emphasized the need for health care that is "culturally safe" in order to overcome power imbalances that shape interactions between health-care providers and Indigenous clients [30]. Culturally safe care simultaneously privileges Indigenous worldviews and acknowledges the impact of structural violence experienced by colonized peoples in health-care settings [31]. Indigenous experts also recognize the importance of "culture as intervention" to support resilience and resistance to HIV infection as well as traumainformed counseling as a response to the harmful effects of historical and lifetime trauma [1]. Others have noted the critical role of interconnectedness with family and community to support health and healing among Indigenous individuals [32]. Further research has highlighted the importance of non-medical, supplementary support for young Indigenous people in order to break through layers of marginalization experienced in their everyday lives [33].

Mobile health (mHealth), the provision of health care via mobile phones, has been identified as potential tool to support young drug-using Indigenous people to overcome barriers to HIV prevention. Recent trials in subSaharan Africa have provided evidence that supportive text-message interventions can improve HIV treatment adherence and viral suppression among people living with HIV $[34,35]$. Others have begun to investigate the impact of mHealth for HIV care and treatment among people living with HIV who use drugs [36-38]. However, 
much of the existing evidence on mHealth interventions for HIV prevention has focused on improving HIV knowledge by delivering HIV prevention messages to diverse at-risk groups [39]. To our knowledge, no studies have investigated the potential for a two-way supportive text-message program to reach out to young drug-using Indigenous people to reduce vulnerability to HIV infection.

This protocol outlines a multi-site Zelen pre-randomized trial to measure the effect of a culturally safe, two-way supportive text-message intervention to reduce HIV vulnerability among young Indigenous people who use illicit drugs. This work will build on findings from recent studies implementing mHealth interventions in HIV clinics in Nairobi, Kenya and Vancouver, Canada [34, 40]. Outcomes include HIV propensity score, HIV risk, resilience, psychological distress, access to drug-related services, and connection to culture. We hypothesize that the Cedar Project WelTel mHealth intervention will result in improved uptake and access to HIV prevention services, including health-care, harm reduction and addictions services, cultural supports, housing, and counseling. We believe that, together with the supportive element that "someone cares" enough to check in each week, improved access to services will help strengthen participants' resilience and reduce vulnerability to HIV infection.

\section{Methods and design Study design}

The Cedar Project WelTel mHealth study is a two-site, two-arm, parallel group, open, stratified Zelen prerandomized controlled trial to measure the effect of a culturally safe, two-way supportive text-message intervention to reduce HIV vulnerability among young Indigenous people who use illicit drugs in a community-based setting (ClinicalTrials.gov identifier NCT02437123). Participants will be pre-randomized to two groups-either the mHealth intervention or standard care-at a 1:1 allocation ratio.

\section{Study setting}

This trial is a sub-study nested within the Cedar Project, a cohort study of young Indigenous people who use injection and non-injection drugs and reside in Vancouver and Prince George, BC, Canada. Initial recruitment into the Cedar Project occurred between 2003 and 2007 and was re-opened in 2011. To date, 738 participants have enrolled in the main Cedar Project cohort in Vancouver and Prince George. Eligibility criteria included being between the ages of 14 to 30 at enrollment, provision of informed consent, self identification as having Indigenous ancestry, and smoking or injecting drugs-including crystal methamphetamine, opiates, crack or cocaine-at enrollment. Cedar Project participants complete an enrollment visit as well as follow-up visits every 6 months.
At each study visit, participants complete detailed questionnaires eliciting demographic, behavioural and health information, administered by trained Indigenous interviewers. Participants also provide a venous blood sample for HIV and hepatitis $\mathrm{C}$ virus antibody testing. In gratitude for participants' time, honoraria are provided at each follow-up visit. The Cedar Project has been approved by the University of British Columbia Providence Health Care Research Ethics Board (H02-50304).

The Cedar Project study storefront research offices are located in Vancouver's downtown eastside and in the downtown core of Prince George. Vancouver is a large city in southern $\mathrm{BC}$ and is located on the traditional territory of the Coast Salish peoples. Prince George is a forestry and mining town in the province's northern interior, located on the traditional territory of the Lheidli T'enneh people. Cedar Project participants represent many of the diverse First Nations, Inuit and Métis communities across Canada and are often living far away from their home communities. Participants are highly transient, moving frequently between cities and reserves, making continuity of care a tremendous challenge [41]. Indigenous interviewers and nurses support both sites and are encouraged to provide referrals to cultural supports, health care, substance use services, food programs, housing, and counseling for participants who seek them.

\section{The Cedar Project Partnership model}

Since its inception, the Cedar Project has been community-driven, interdisciplinary research that responds to the continuing crisis of HIV infection and contributes to the health and healing of young Indigenous people who use drugs. The Cedar Project Partnership, an independent body of Indigenous Elders, health and social service experts, researchers, and elected leaders, governs the entire research process. The Cedar Project Partnership recognizes that conducting culturally safe research with young, vulnerable Indigenous people requires creating safe spaces where their identities, voices and stories are heard and respected [30]. A critical component of the Cedar Project is to ensure that our site offices are safe, welcoming settings without judgement of drug use, where police are not allowed, and cultural identity is honoured. Part of building cultural safety into the Cedar Project includes supporting access to traditional foods and ceremonies, such as through annual feasts, memorials, and a recent Learning Potlatch to honour our partners and participants held in Prince George. Overall, our paradigm is to acknowledge grief and historical trauma while building on young Indigenous peoples' strengths.

\section{Participants}

A stratified random sample of participants in the Cedar Project cohort will be selected for participation in the 
Cedar Project WelTel mHealth study (Fig. 1). A sampling frame of eligible Cedar Project participants will be created on the basis of the following eligibility criteria:

(1) Currently enrolled in the Cedar Project

(2) Completed main Cedar Project baseline questionnaire and attended at least one follow-up visit since 2009

(3) Had not tested positive for HIV

(4) Joined study in Vancouver or Prince George
(5) Alive at initiation of the Cedar Project WelTel mHealth study.

Participants who do not meet the eligibility criteria or who decline to participate will be excluded. Stratified randomization in a 1:1 ratio will be used to ensure a balance of key factors, including study site (Prince George and Vancouver) and reporting injection drug use at the last visit (yes versus no). Sampled participants will be randomly assigned to receive the intervention or be

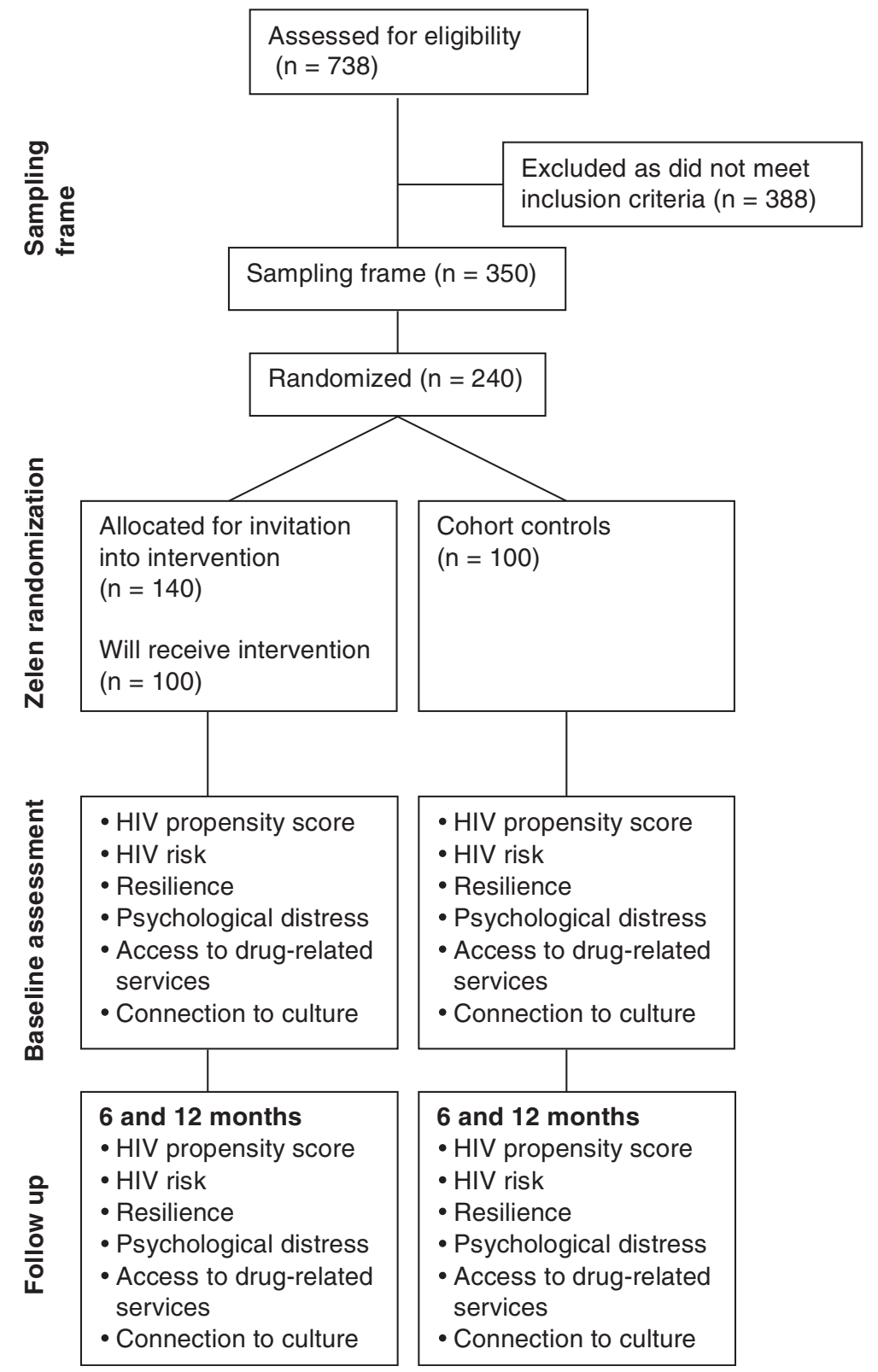

Fig. 1 Cedar Project mHealth Study for HIV prevention CONSORT diagram of study design 
included in the comparison group through computergenerated codes developed by a statistician at the Centre for Evaluation and Outcome Sciences at St. Paul's Hospital. A list of participants to be invited into the intervention arm will be distributed to each study site. Informed consent will be sought from each participant invited to receive the intervention who agreed to participate. Those assigned to the comparison group will continue in the usual Cedar Project cohort study under its existing informed consent with no change whatsoever to their participation in the overall study. Target enrollment is set at 200 participants.

\section{Intervention group}

To ensure that we reach our target sample, initial randomization will be slightly unbalanced to accommodate refusals to participate or difficulty locating participants. A total of 140 participants (70 from each site) will be randomly allocated to receive the invitation to participate in the intervention arm of the mHealth study. This will allow for an estimated 20 participants per site who cannot be reached or who decline to participate. Cedar staff will invite and enroll participants from this list to join the study until enrollment reaches 50 at each study site. Three attempts will be made to invite participants in the intervention arm into the study, including during regular visits to the research offices, phone, email, word of mouth, and street outreach.

\section{Comparison group}

One hundred Cedar Project participants who meet the eligibility criteria will be allocated to serve as the cohort comparison group, stratified on the same variables used to select the intervention group. Participants randomly assigned to the control group will continue as Cedar Project participants under usual circumstances. This group of cohort controls will not be required to provide any additional data to the study beyond that which they provide in semi-annual follow-up interviews as participants in the main Cedar Project study.

\section{The intervention}

The Cedar Project WelTel mHealth intervention being evaluated is a structured mobile-phone initiative to connect young Indigenous people who use drugs with Cedar Case Managers in a community-based setting. The intervention consists of a package of supports, including a mobile phone and cellular plan, weekly two-way text messaging, and support from Cedar Case Managers. The intervention will be implemented over a 16-month period, and all participants will receive the intervention for a minimum of 6 months.

Cedar Case Managers include Indigenous and nonIndigenous nurses and Cedar Project staff members who have extensive frontline outreach experience with young Indigenous people who use drugs in Prince George and
Vancouver. Cedar Case Managers follow a "culturally safe" approach that acknowledges both trauma and strengths and includes an explicit focus on the critical roles of cultural assets and safe relationships with care providers in HIV risk reduction [31].

Participants allocated to receive the intervention will be provided with a cellular phone at the start of the study. Each phone is pre-programmed with several phone numbers for emergency and health-related services relevant to the study site. During the study period, participants will receive a monthly cellular plan that includes unlimited calling and texting within Canada, but no data. We made an explicit decision to include longdistance calling and texting to provide the opportunity for participants to connect with family and loved ones living in home communities and elsewhere in Canada. If phones are lost or stolen, participants will be eligible to receive one replacement. If two phones are lost, the participant may bring their own sim card-enabled cellular handset to use with the study monthly cellular plan.

The supportive text-message component of the study is based on the intervention tested in the WelTel Kenya1 trial (Fig. 2) [34]. Each Monday at noon, a text message saying, "How's it going?" is automatically sent to intervention arm participants through the WelTel mHealth software platform. Cedar Case Managers will login within 24 to 48 hours to triage the incoming text messages. They will respond to all participants and follow up with participants who replied with a specific problem or need. On Wednesday, participants who have not replied will receive an additional text saying, "Haven't heard from you, are you OK?" On Thursday or Friday, staff will attempt to call all remaining participants who have not

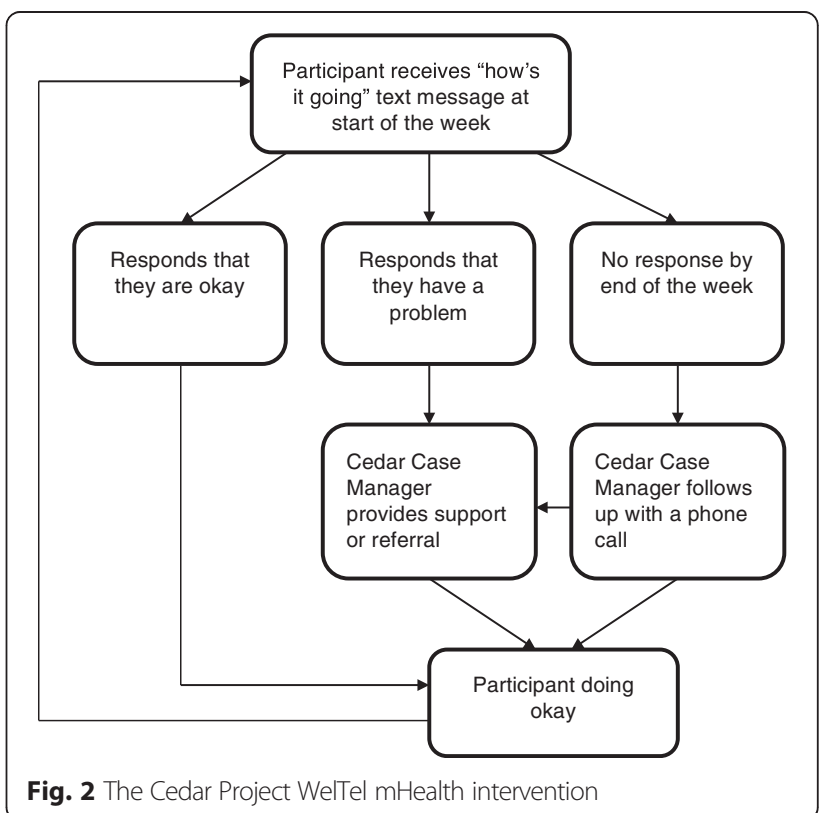


responded by text message. Throughout, study staff will keep a $\log$ of all responses and actions taken through the WelTel platform.

\section{Blinding}

We recognize that blinding in a trial such as ours is challenging. Care providers (Cedar Case Managers) and intervention arm participants cannot be blinded, as the mHealth intervention requires overt participation and an "invite list" is provided upfront. However, participants in both the intervention and control groups will be blinded to specific study hypotheses. Primary and secondary outcomes will be drawn from routine data collection that occurs as part of participation in the overall cohort; it is unlikely that participants will identify the specific questions used to generate outcomes from our extensive questionnaires. The data analyst will also be blinded to group allocation.

\section{Objectives}

The study's primary objective is to test whether the Cedar Project mHealth intervention reduces vulnerability to HIV among young Indigenous people who use drugs, measured by using an HIV propensity score described below. Our secondary objectives are to test the specific ways the mHealth intervention may reduce vulnerability to HIV by exploring HIV risk behaviours, resilience, psychological distress, access to drug-related services, and connection to culture among participants over the course of the study.

We hypothesize that participants receiving the intervention will seek help from Cedar Case Managers to navigate barriers to drug-related services, housing, cultural supports, and health care. Also, we hypothesize that participants will use the phones as tools to connect with other supports and sources of strength in their lives, including family, work, culture, and services. Thus, we hypothesize that the intervention will help young people to work towards goals related to their own health and well-being, supporting them to reduce HIV risk.

\section{Outcome measures}

\section{Primary outcomes}

The primary outcome (Table 1) is HIV risk, measured by an HIV propensity score assessed at the 6-month time point. Previous analyses of Cedar Project data with respect to HIV infection have identified several factors as associated with HIV infection, including recent injection drug use, high-frequency drug use, needle sharing, and participation in sex work [12]. Logistic regression of risk factors for prevalent HIV infection at study recruitment will provide coefficients to combine these four risk variables into a single primary outcome measuring propensity for HIV infection. Mean change in HIV propensity score from baseline to 6 months will be used to determine the impact of the intervention on HIV vulnerability.

\section{Secondary outcomes}

The potential benefits of the supportive mHealth intervention are broad. As a result, multiple secondary outcomes are of interest. These were selected through consultation with academic and community partners and are part of routine Cedar Project data collection. They fall under five categories: HIV risk, resilience, psychological distress, access to drug-related services, and connection to culture. For all secondary outcomes, the effect of the mHealth intervention will be measured as change in the outcome from baseline to 6 months. Participants with sufficient follow-up time will contribute to analyses of all outcomes at the 1-year time point to determine longer-term effects of the intervention.

HIV propensity (12 months) Participants who received the intervention for a sufficient length of time will contribute to a secondary analysis of change in HIV propensity at 1 year to determine longer-term effects of the Cedar Project WelTel mHealth intervention. The HIV propensity variable will be generated by using the same approach used for the primary outcome.

HIV vulnerability Although the effect of the intervention on HIV risk will be assessed in the primary analysis, we will also analyze the effect of the intervention on specific behaviours. Several self-reported binary measures will be used to determine the effect of the intervention on HIV vulnerability in the previous 6-month period, including injection drug use in the past 6 months, high-frequency (daily or more) drug use, needle sharing, and participation in sex work. These measures will be ascertained from Cedar Project questionnaires collected every 6 months as part of the main Cedar Project cohort. HIV vulnerability will be assessed at baseline, 6 months, and 1 year.

Resilience Resilience, or the ability to cope with adversity, will be characterized by using the Connor-Davidson Resilience Scale (CD-RISC). The creators of the scale broadly define resilience as "personal qualities that enable one to thrive in the face of adversity" [42]. The CDRISC scale measures resilience via 25 items on a fivepoint scale with scores ranging between 0 and 100, with higher scores indicating greater resilience. Resilience will be assessed at baseline, 6 months, and 1 year.

Psychological distress The Symptom Checklist-90-R (SCL-90-R) is a 90-item self-reported symptom inventory that measures the severity of nine dimensions of psychological distress in the past 3 months scored on a five-point Likert scale (from 'not at all' to 'extremely'). Participants' 
Table 1 Outcome measures

\begin{tabular}{|c|c|c|c|c|c|}
\hline Outcome measure & Specific variable & Analysis metric & $\begin{array}{l}\text { Hypothesis } \\
\text { (intervention > control) }\end{array}$ & Type & Analysis \\
\hline \multicolumn{6}{|l|}{ Primary } \\
\hline $\begin{array}{l}\text { HIV propensity } \\
\text { ( } 6 \text { months) }\end{array}$ & HIV propensity score & Mean change - HIV propensity score & Decrease in HIV risk & Continuous & T test \\
\hline \multicolumn{6}{|l|}{ Secondary } \\
\hline $\begin{array}{l}\text { HIV propensity } \\
\text { (12 months) }\end{array}$ & HIV propensity score & Mean change - HIV propensity score & Decrease in HIV risk & Continuous & T test \\
\hline \multirow[t]{4}{*}{ HIV vulnerability } & Injection drug use & Mean change - proportion injection & Decrease in injection & Binary & $x^{2}$ test \\
\hline & High-frequency injection & $\begin{array}{l}\text { Mean change - proportion frequent } \\
\text { injection }\end{array}$ & $\begin{array}{l}\text { Decrease in injection } \\
\text { frequency }\end{array}$ & Binary & $x^{2}$ test \\
\hline & Needle sharing & Mean change - proportion needle sharing & $\begin{array}{l}\text { Decrease in needle } \\
\text { sharing }\end{array}$ & Binary & $x^{2}$ test \\
\hline & Participation in sex work & Mean change - proportion sex work & Decrease in sex work & Binary & $x^{2}$ test \\
\hline Resilience & $\begin{array}{l}\text { Connor-Davidson Resilience } \\
\text { Scale (CD-RISC) }\end{array}$ & Mean change - CD-RISC score & Increase in resilience & Continuous & T test \\
\hline Psychological distress & $\begin{array}{l}\text { Symptom Checklist-90-R } \\
\text { (SCL-90-R) }\end{array}$ & Mean change - SCL-90-R score & $\begin{array}{l}\text { Decrease in } \\
\text { psychological distress }\end{array}$ & Continuous & T test \\
\hline \multirow[t]{5}{*}{$\begin{array}{l}\text { Access to drug- } \\
\text { related services }\end{array}$} & $\begin{array}{l}\text { Opioid substitution therapy } \\
\text { (OST) }\end{array}$ & Mean change - proportion accessed OST & Increase in access OST & Binary & $x^{2}$ test \\
\hline & Needle exchange (NX) & Mean change - proportion attended NX & Increase in access NX & Binary & $x^{2}$ test \\
\hline & Safe injection facility (SIF) & Mean change - proportion visited SIF & Increase in access SIF & Binary & $x^{2}$ test \\
\hline & Drug treatment & $\begin{array}{l}\text { Mean change - proportion seeking } \\
\text { treatment }\end{array}$ & $\begin{array}{l}\text { Increase in seeking } \\
\text { treatment }\end{array}$ & Binary & $x^{2}$ test \\
\hline & Tried to quit using drugs & Mean change - proportion trying to quit & $\begin{array}{l}\text { Increase in trying to } \\
\text { quit }\end{array}$ & Binary & $x^{2}$ test \\
\hline \multirow[t]{2}{*}{$\begin{array}{l}\text { Connection to } \\
\text { culture }\end{array}$} & $\begin{array}{l}\text { Participation in traditional } \\
\text { ceremonies }\end{array}$ & $\begin{array}{l}\text { Mean change - proportion participated in } \\
\text { traditional ceremony }\end{array}$ & $\begin{array}{l}\text { Increase in access to } \\
\text { ceremony }\end{array}$ & Binary & $x^{2}$ test \\
\hline & $\begin{array}{l}\text { Frequently living by traditional } \\
\text { culture }\end{array}$ & $\begin{array}{l}\text { Mean change - proportion lived by } \\
\text { traditional culture }\end{array}$ & $\begin{array}{l}\text { Increase in traditional } \\
\text { culture }\end{array}$ & Binary & $x^{2}$ test \\
\hline
\end{tabular}

All variables refer to behaviour in the 6-month period preceding the interview. All variables are measured as mean change in proportion or score from baseline

SCL-90-R scores will be transformed into an average Global Severity Index, providing a single average measure that profiles overall degree of psychological distress [43]. Psychological distress will be assessed at baseline, 6 months, and 1 year.

Access to drug-related services Self-reported access to drug-related services, including opioid substitution therapy, needle exchange, safe injection facility, and drug treatment, in the previous 6-month period, will be ascertained from the main Cedar Project questionnaire. Proportions of participants reporting access to these services will be compared in the intervention and control groups. We will also determine whether there are differences among treated and control groups in terms of proportion of people who tried to quit in the previous 6-month period. Access to drug-related services will be assessed at baseline, 6 months, and 1 year.

Connection to culture Connection to Indigenous culture has been hypothesized as a key protective factor for young Indigenous people who use drugs. It will be assessed by using two dichotomous variables that measure cultural activity in the prior 6-month period: (1) self-reported participation in traditional ceremonies (including potlatch, feast, fast, burning ceremony, washing ceremony, naming ceremony, big/smoke house, rites of passage, smudge, dances, or any other traditional Indigenous ceremony) and (2) frequency living by traditional culture (never/rarely versus often/always). These variables were defined by Earl Henderson (Cree, Métis) and Violet Bozoki (Lheidli T'enneh) who are Indigenous Elders, traditional knowledge keepers, and members of the Cedar Project Partnership.

\section{Sample size}

Considering current follow-up rates, we are likely able to reach and recruit at least 200 participants for participation in both arms of the Cedar Project WelTel mHealth study. As mentioned previously, the primary objective is HIV risk, measured by using an HIV propensity score evaluated at 6 months. On the basis of a 1:1 allocation ratio, a 
significance level of 0.05 (one-sided) and $80 \%$ power, it is estimated that we require 78 participants in each study arm. This computation assumes a standardized mean effect size (using Cohen's d) of 0.40. Assuming attrition rates of approximately $20 \%$, we intend to over-enroll, resulting in a target of 200 participants overall (100 in each arm). Sample size was calculated by using the pwr package in R, software version 3.2.1.

\section{Statistical analysis}

The analysis and reporting of the results will follow the CONSORT (Consolidated Standards of Reporting Trials) guidelines [44]. Descriptive statistics of socio-demographic, historical/lifetime trauma, sex- and drug-related HIV vulnerabilities, and health-related variables will be presented to assess comparability of the intervention and control groups. Mean (standard deviation) or median (quartiles) will be used for continuous variables and count (percentage) for categorical variables. Our primary analysis to evaluate the effect of the mHealth intervention on HIV prevention will be by intention to treat (ITT) for primary and secondary outcomes among all randomly assigned participants according to the study group to which they were originally allocated. Student's $t$ tests and chi-squared tests will be used to determine differences in the mean change in proportions or scores from baseline at 6 months or 1 year between the two study arms. If baseline characteristics are found to be substantially different between the two study arms, we will adjust for these factors by using regression models. In addition, we will conduct a secondary analysis by using a modified ITT approach including only participants who agreed to participate in the intervention making up the intervention group (excluding over-sampled participants who did not receive the intervention). To minimize the risk of bias introduced in our modified ITT analysis, we propose to use complier average causal effect (CACE) analysis [45]. This approach will allow us to retain the initial random assignment by taking into account two subgroups within the group pre-randomized to receive the intervention (those who did and did not actually receive the intervention) and then helping us to identify a similar subgroup of control participants who could have been expected to receive the intervention had it been offered [45]. By comparing expected and observed outcomes in these subgroups, we will obtain a less biased estimate of effect of the intervention [45]. Missing data will be approached by using multiple imputation. An up-to-date version of $\mathrm{R}$ statistical software will be used to conduct all analyses [46]. All tests will be two-sided; $P$ values of less than 0.10 will be considered significant. We plan to conduct four predetermined exploratory subgroup analyses that may help to tailor the intervention for specific high-need populations, including comparisons by gender, city, injection drug use, and urban versus rural. Statistical methods similar to those described above for whole-group analyses will be conducted for primary and secondary outcomes. All subgroup results will be reported, regardless of significance. Inferences will be hypothesis-generating, given concerns related to multiple comparisons and lower power to detect an effect in subgroups.

\section{Nested studies \\ Study implementation}

As this study is the first of its kind in a communitybased setting among young Indigenous people who use drugs, it is critical that the implementation of the intervention be described in detail. Daily mHealth memos prepared by each study site, combined with procedural documents, will inform a description of how a supportive two-way text-message program looks and functions in practice. Descriptive statistics generated from the mHealth baseline questionnaire will provide additional insight into the feasibility of applying this program in similar settings. The survey asks about ownership and use of mobile phones, how often participants text message, and perceived helpfulness or concerns about receiving text messages related to health.

\section{Types of support requested}

Examining the types of support requested by Cedar Project WelTel mHealth participants via text will help to identify current challenges accessing prevention services and support among young Indigenous people who use drugs. A comparative content analysis of text-message interactions captured on the WelTel platform over the study period will be used for this purpose. This descriptive analysis will help inform scale-up of this program, including the human resource requirements for future implementation in similar settings.

\section{Participant perceptions}

Participant perceptions of the mHealth intervention will be evaluated via the mHealth follow-up questionnaire. All intervention arm participants will be invited to complete an administered questionnaire to assess satisfaction with the care and support they received via the intervention. Evaluation of patient perceptions will include thematic analysis of narrative responses and basic descriptive statistics.

\section{Ethics}

The Cedar Project follows the guidelines provided in the Tri-Council Policy Statement on Ethical Conduct for Research Involving Humans - Chapter Nine: Research involving the First Nations Inuit and Métis Peoples of Canada [47]. In addition, the study will adhere to the principles of Ownership, Control, Access and Possession in relation to research with Indigenous people [48]. Through the Cedar 
Project Partnership, Indigenous collaborators will continue to be involved in the conception, design and interpretation of the results of the Cedar Project WelTel mHealth study. The Cedar Project Partnership will serve as the data and safety monitoring board for this study. They have also approved this manuscript for publication. The Cedar Project WelTel mHealth study has been approved by the University of British Columbia Providence Health Care Research Ethics Board (H13-02718), and we will be accountable to them for approval and monitoring.

All participants have provided informed consent as part of the main Cedar Project cohort study. Participants randomly assigned to receive an invitation to participate in the Cedar Project WelTel mHealth intervention will undergo an additional consent process. After the study participant is invited to participate in the Cedar Project WelTel mHealth study, a trained Cedar Project staff member will describe the study. If the person would like to enroll, the research staff will review the consent form and answer any questions. Participants who give consent are provided with a copy of the study introduction letter and consent form.

\section{Harms}

Adverse events will be documented in writing and reported to investigators. Study staff at both sites have been trained in recognizing and reporting of adverse events, including those directly attributable to the intervention (e.g., accidental disclosure of illicit drug use) and those resulting from trial participation. Because the study takes place in a community-based (not clinical) setting, staff will make referrals to care where appropriate. Potential harms will be outlined to participants during the informed consent process. All unanticipated risks to human participants or others will be reported to the Cedar Project Partnership and the University of British Columbia/Providence Health Care Research Ethics Board where appropriate.

\section{Dissemination}

Knowledge translation is a hallmark of the Cedar Project and will be a key component of the Cedar Project WelTel mHealth study. Integrated knowledge translation is planned throughout the study, and updates will be provided to the Cedar Project Partnership at quarterly meetings. Findings will be shared through ceremony with Indigenous partners; peer-reviewed publications and presentations; and consultations with policy makers and service delivery organizations to support uptake of results.

\section{Discussion}

Innovative, culturally safe interventions that address the barriers to HIV prevention while supporting the strength of young Indigenous people who use drugs are urgently needed. This study, due to report its findings in 2017, tests the effectiveness of a two-way supportive textmessage program delivered in a community-based setting to support HIV prevention among young Indigenous people who use drugs. Despite presenting a tremendous opportunity to connect young, highly transient Indigenous people who use drugs to prevention services and support, supportive two-way mHealth programs have yet to be tested for HIV prevention in a community-based setting with young Indigenous people who use drugs [34, 49-51].

Other researchers have used two-way supportive messaging interventions to engage people living with HIV in care and to improve adherence [34, 36, 40, 52, 53]. Recent randomized controlled trials in Kenya indicated that introducing mobile-phone technology into HIV case management improved HIV treatment adherence and HIVrelated clinical outcomes, despite high levels of poverty, remote and rural living, transience, stigma, and discrimination [34, 50, 54]. These studies have demonstrated the importance of text-message programs that are both supportive and interactive [35, 55-57], as passive mHealth programs that seek to monitor, remind or educate have had limited success [58-60]. It is important to note that we have deliberately chosen an open-ended weekly text message to allow participants to set their own priorities around their health and well-being, as well as to avoid possible disclosure of drug use and other sensitive issues.

We have chosen a cohort-embedded Zelen prerandomized controlled trial design [61-63]. Our ongoing Cedar Project cohort study provides the opportunity to support recruitment into the embedded trial and also allows us to use routinely measured outcomes collected longitudinally among the whole cohort. All participants entering the cohort have consented to providing observational data. Consent to "try" the intervention is being sought only from those who have been pre-randomized to receive the intervention [63]. Study outcomes among randomly selected participants will be compared with those of the cohort members not randomly selected to receive the intervention. Another advantage of this design is to minimize false hopes, resentment, and contamination among controls had they learned they were not receiving a potentially valued intervention through trial consent and randomization [61, 62]. In our instance, as it is not possible to completely separate intervention participants from the rest of the cohort, it is likely that some members of the cohort will come to know about the intervention. However, even if control participants have or obtain a phone and cellular plan, full crossover of participants from control to intervention is not possible, owing to the nature of the intervention. As noted above, neither group will be aware of the hypotheses under study. A limitation of this design is that a significant number of participants allocated to the intervention arm may refuse to receive it whereas control subjects do not have this option. Including the former 
group in an ITT analysis may dilute the treatment effect, whereas excluding them could lead to a biased comparison. In our case, we believe that the intervention is likely to be highly valued among invitees and there will be few who refuse. Nevertheless, this will be monitored closely, and as noted earlier, we will also use a modified ITT approach.

A key component of the Cedar Project WelTel mHealth study is relational accountability [64]. We honour our relationships with study participants who have continued to share their stories with us since joining the Cedar Project. Testing this intervention is a way for us to respond to the clear message from Cedar Project participants about the importance of having support to navigate barriers to care and services that they face in their daily lives. An additional component of our relational accountability is through the governing leadership of the Cedar Project Partnership, who work to ensure that this research is relevant to the communities they represent and is conducted in a good way.

\section{Limitations}

Our decision to over-sample in the intervention arm to adjust for refusals to participate or difficulty locating participants may result in a dilution of the effect of the intervention in ITT analysis. As a result, we have proposed a parallel, modified ITT analysis, which may be affected by selection bias. Diversity of HIV risk among participants (non-homogeneity) may also dilute the detected effect of the intervention. Representativeness of the sample is dependent on representativeness of the Cedar Project cohort study sample overall; however, we have made a considerable attempt to ensure that the sample represents the population under study [12]. As this study involves a package of support that includes mobile phones, two-way text messaging, and support, it is difficult to determine the relative contribution of components of the package to any effect the intervention may have. The qualitative nested studies will help in this regard. Furthermore, we cannot rule out that simultaneous co-interventions could impact our results. As BC's HIV incidence has been stable for several years and many public health initiatives (including needle exchanges and safe injection sites) are already in place, we feel it is unlikely that new transformative co-interventions will take effect over the study period. However, we will monitor potential co-interventions that could impact study outcomes through our main cohort questionnaires, as well as track major policy and programmatic changes in the Province. Blinding in this study is not possible as assessors have strong relationships with participants. In addition, loss to follow-up may be higher in the comparison group than the intervention group. Cedar Project participants are highly transient and often difficult to track down [41]. Having a mobile phone and consistent airtime will likely make it easier to reach participants receiving the intervention for follow-up visits. Active follow-up with all participants through phone, email, and outreach will help ensure retention and interview appointment attendance. In addition, our measures may not adequately capture complex concepts such as connection to culture and resilience. Finally, the intervention under study is complex and multifaceted. It may be that our proposed outcomes do not adequately capture the impact of the intervention on the lives of participants involved.

\section{Trial status}

Enrollment in the trial has begun but has not yet reached full enrollment.

\section{Abbreviations}

BC: British Columbia; CD-RISC: Connor-Davidson resilience scale; ITT: Intention to treat; mHealth: Health care delivered via mobile phone; NX: Needle exchange; OST: Opioid substitution therapy; SIF: Safe injection facility; SCL-90-R: Symptom checklist 90-R.

\section{Competing interests}

RTL is the co-founder and executive director of the WelTel International mHealth Society and has an interest in WelTel, the company that provides the SMS software platform. The other authors declare that they have no competing interests.

\section{Authors' contributions}

$\mathrm{KJ}$ helped to conceive of the study, develop the study design, secure the funding, develop study procedures, initiate study implementation, write the study protocol and is a grant holder. LD helped to conceive of the study, develop the study design and provide input on Indigenous research methods and cultural safety in study protocols and procedures. RTL helped to conceive of the study and develop the study design. PMS helped to conceive of the study, develop the study design, secure the funding, develop study procedures, initiate study implementation and is a grant holder. MTS helped to conceive of the study, develop the study design, secure the funding and is a grant holder. MEP helped to secure the funding and write the study protocol. AJF helped to secure the funding, develop study procedures, initiate study implementation, and write the study protocol. VT helped to develop study procedures, initiate study implementation and provide input on Indigenous research methods and cultural safety in study protocols and procedures. MLVDK helped to write the study protocol and gave input on study design, measures and statistical analyses. SP provided input on Indigenous research methods and cultural safety in study protocols and procedures. The Cedar Project Partnership helped to conceive of the study, develop the study design, secure the funding and provided governance and leadership. All authors contributed critical intellectual input to refinement of the study protocol and approved the final manuscript.

\section{Acknowledgements}

The authors would like to thank the Cedar Project participants who continue to share their voices with us. We remain grateful to the Cedar Project Partners, including Elders Violet Bozoki (Lheidli T'enneh) and Earl Henderson (Cree, Métis); Prince George Native Friendship Centre; Carrier Sekani Child \& Family Services; Splatsin Secwepmc Nation; Adams Lake Indian Band; Neskonlith Indian Band; Positive Living North; Red Road Aboriginal AIDS Network; Canadian Aboriginal AIDS Network; Vancouver Native Health Society; Central Interior Native Health; and All Nations Hope Network, who guide and govern this study. Thanks to the Cedar Project staff (including Vicky Thomas, Amanda Wood, Sharon Springer, Matt Quenneville, Jill Fikowski and Shawna Morrison) for their invaluable contributions. Thank you also to the Oak Tree and WelTel staff who provided mentoring and shared best practices as we initiated the Cedar Project WelTel mHealth study. We would like to acknowledge the support of TELUS for providing mobile phones and subsidized monthly cellular plans and of WelTel for contributing their mHealth patient engagement software. 


\section{Funding}

The Cedar Project WelTel mHealth study is supported by a Canadian Institutes for Health Research (CIHR) operating grant (RN 156 278-272441) with additional funding from the St. Paul's Hospital Foundation Enhanced Patient Care Fund. The funding sources had no role in the design of the study and will not have any role in its execution, analyses, interpretation, or decision to submit results. $\mathrm{KJ}$ is supported by a CIHR Doctoral Award (May 2013).

\section{Author details}

${ }^{1}$ School of Population and Public Health, Faculty of Medicine, University of British Columbia, 2206 East Mall, Vancouver, BC V6T 1Z3, Canada. ${ }^{2}$ The Cedar Project, Centre for Health Evaluation and Outcome Sciences, St. Paul's Hospital, 588-1081 Burrard Street, Vancouver, BC V6Z 1Y6, Canada. ${ }^{3}$ Department of Public Health Sciences, Karolinska Institutet, Tomtebodavägen 18a, Campus Solna, Stockholm 171 77, Sweden. ${ }^{4}$ Division of Infectious Diseases, Faculty of Medicine, University of British Columbia, 566-828 West 10th Avenue, Vancouver, BC V5Z 1L8, Canada. ${ }^{5}$ Vancouver Native Health Society, 455 Hastings Street E, Vancouver, BC V6A 1P5, Canada. ${ }^{6}$ Canadian Aboriginal AIDS Network, 6520 Salish Drive, Vancouver, BC V6N 2C7, Canada. ${ }^{7}$ Neglected Global Diseases Initiative, Faculty of Medicine, University of British Columbia, 564-828 West 10th Avenue, Vancouver, BC V5Z 1L8, Canada.

Received: 29 July 2015 Accepted: 23 February 2016 Published online: 09 March 2016

\section{References}

1. Simoni JM, Sehgal S, Walters KL. Triangle of risk: urban American Indian women's sexual trauma, injection drug use, and HIV sexual risk behaviors. AIDS Behav. 2004;8:33-45.

2. Duncan KC, Reading C, Borwein AM, Murray MC, Palmer A, Michelow W, et al. HIV incidence and prevalence among Aboriginal peoples in Canada. AIDS Behav. 2011;15:214-27.

3. Negin J, Aspin C, Gadsden T, Reading C. HIV Among Indigenous peoples: a review of the literature on HIV-related behaviour since the beginning of the epidemic. AIDS Behav. 2015;19:1720-34.

4. Christian WM, Spittal PM. The Cedar Project: acknowledging the pain of our children. Lancet. 2008;372:1132-3.

5. Chansonneuve D. Addictive behaviours among Aboriginal people in Canada. Ottawa: Aboriginal Healing Foundation; 2007.

6. Duran E, Duran B, Brave Heart MY, Yellow H-DS. Healing the American Soul Wound. In: Danieli Y, editor. International Handbook of Multigenerational Legacies of Trauma. New York: Plenum Press; 1998. p. 341-54.

7. Brave Heart MY. The historical trauma response among natives and its relationship with substance abuse: a Lakota illustration. J Psychoactive Drugs. 2003;35:7-13.

8. Truth \& Reconcilliation Commission of Canada. Honouring the Truth, Reconciling for the Future: Summary of the Final Report of the Truth and Reconciliation Commission of Canada. Winnipeg: TRC; 2015

9. Chansonneuve D. Reclaiming connections: understanding residential school trauma among Aboriginal people. Ottawa: Aboriginal Healing Foundation; 2005.

10. Blackstock C. First Nations child and family services: restoring peace and harmony in First Nations communities. In: Kufeldt K, McKenzie B, editors. Child welfare: connecting research policy and practice. Waterloo: Wilfred Laurier University Press; 2003. p. 331-43.

11. Clarkson AF, Christian WM, Pearce ME, Jongbloed KA, Caron NR, Teegee MP, et al. The Cedar Project: negative health outcomes associated with involvement in the child welfare system among young Indigenous people who use injection and non-injection drugs in two Canadian cities. Can J Public Health. 2015;106:e265-70.

12. Spittal PM, Craib KJ, Teegee M, Baylis C, Christian WM, Moniruzzaman A, et al. The Cedar project: prevalence and correlates of HIV infection among young Aboriginal people who use drugs in two Canadian cities. Int J Circumpolar Health. 2007;66:226-40

13. Pearce ME, Christian WM, Patterson K, Norris K, Moniruzzaman A, Craib KJP, et al. The Cedar Project: historical trauma, sexual abuse and HIV risk among young Aboriginal people who use injection and non-injection drugs in two Canadian cities. Soc Sci Med. 2008;66:2185-94.

14. Barlow JK. Examining HIV/AIDS among the Aboriginal population in Canada in the post-residential school era. Ottawa: Aboriginal Healing Foundation; 2003.
15. Pearce ME, Jongbloed K, Richardson C, Henderson E, Pooyak S, Oviedo-Joekes $\mathrm{E}$, et al. The Cedar Project: resilience in the face of HIV vulnerability within a cohort study involving young Indigenous people who use drugs in three Canadian cities. BMC Public Health. 2015;15: 1095-106.

16. Public Health Agency of Canada. Population-Specific HIV/AIDS Status Report: Aboriginal Peoples. Ottawa: PHAC; 2010.

17. Public Health Agency of Canada. HIV and AIDS in Canada: Surveillance Report to December 31, 2013. Ottawa: PHAC; 2014.

18. Miller CL, Strathdee SA, Spittal PM, Kerr T, Li K, Schechter MT, et al. Elevated rates of HIV infection among young Aboriginal injection drug users in a Canadian setting. Harm Reduct J. 2006;3:9.

19. Dell CA, Lyons T. Harm reduction policies and programs for persons of Aboriginal descent. Ottawa: Canadian Centre on Substance Abuse; 2007.

20. Canadian Aboriginal AIDS Network. Joining the Circle: An Aboriginal Harm Reduction Model. Vancouver, BC: Canadian Aboriginal AIDS Network; no date.

21. Mill J, Edwards N, Jackson R, Austin W, MacLean L, Reintjes F. Accessing health services while living with HIV: intersections of stigma. Can J Nurs Res. 2009;41:168-85.

22. Browne AJ, Fiske J-A. First Nations women's encounters with mainstream health care services. West J Nurs Res. 2001;23:126-47.

23. Allan B, Smylie J. First Peoples, second class treatment: The role of racism in the health and well-being of Indigenous peoples in Canada. Toronto: The Wellesley Institute; 2015.

24. Pinkerton SD. How many HIV infections are prevented by Vancouver Canada's supervised injection facility? Int J Drug Policy. 2011;22:179-83.

25. Gowing L, Farrell MF, Bornemann R, Sullivan LE, Ali R. Oral substitution treatment of injecting opioid users for prevention of HIV infection. Cochrane Database Syst Rev. 2011;8:1-116.

26. Yang J, Oviedo-Joekes E, Christian KWM, Li K, Louie M, Schechter M, et al. The Cedar Project: Methadone maintenance treatment among young Aboriginal people who use opioids in two Canadian cities. Drug Alcohol Rev. 2011;30:645-51

27. Kerr T, Marsh D, Li K, Montaner J, Wood E. Factors associated with methadone maintenance therapy use among a cohort of polysubstance using injection drug users in Vancouver. Drug Alcohol Depend. 2005:80:329-35.

28. Oppal WT. Forsaken: The Report of the Missing Women Commission of Inquiry. Vancouver: Missing Women Commission of Inquiry; 2012.

29. Pearce ME, Blair AH, Teegee M, Pan SW, Thomas V, Zhang H, et al. The Cedar Project: historical trauma and vulnerability to sexual assault among young Aboriginal women who use illicit drugs in two Canadian cities. Violence Against Women. 2015;21:313-29.

30. Papps E, Ramsden I. Cultural safety in nursing: The New Zealand experience. International J Qual Health Care. 1996:8:491-7.

31. Smye V, Browne A. 'Cultural safety' and the analysis of health policy affecting aboriginal people. Nurse Res. 2002;9:42.

32. McCormick RM. Healing through interdependence: the role of connecting in First Nations healing practices. Can J Couns. 1997;31:172-84.

33. Prentice T, Mill J, Archibald CP, Sommerfeldt S, Worthington C, Jackson R, et al. Aboriginal youth experiences of accessing HIV care and treatment. J HIV AIDS Soc Ser. 2011;10:395-413.

34. Lester RT, Ritvo P, Mills EJ, Kariri A, Karanja S, Chung MH, et al. Effects of a mobile phone short message service on antiretroviral treatment adherence in Kenya (WelTel Kenya1): a randomised trial. Lancet. 2010;376:1838-45.

35. Mbuagbaw L, van der Kop ML, Lester RT, Thirumurthy H, Pop-Eleches C, Ye C, et al. Mobile phone text messages for improving adherence to antiretroviral therapy (ART): an individual patient data meta-analysis of randomised trials. BMJ Open. 2013;3, e003950.

36. Ingersoll K, Dillingham R, Reynolds G, Hettema J, Freeman J, Hosseinbor S, et al. Development of a personalized bidirectional text messaging tool for HIV adherence assessment and intervention among substance abusers. I Subst Abuse Treat. 2014;46:66-73.

37. Reback C. Using text messaging to reduce methamphetamine use and sexual risk behaviors and increase ART adherence among men who have sex with men. J Mob Technol Med. 2014;3:8

38. Montoya JL, Georges S, Poquette A, Depp CA, Atkinson JH, Moore DJ. Refining a personalized mHealth intervention to promote medication adherence among HIV+ methamphetamine users. AIDS Care. 2014:26:1477-81.

39. Catalani C, Philbrick W, Fraser H, Mechael P, Israelski DM. mHealth for HIV treatment \& prevention: a systematic review of the literature. Open AIDS J. 2013;7:17 
40. Smillie K, Van Borek N, Abaki J, Pick N, Maan EJ, Friesen K, et al. A qualitative study investigating the use of a mobile phone short message service designed to improve HIV adherence and retention in care in Canada (WelTel BC1). J Assoc Nurses AIDS Care. 2014;25:614-25.

41. Jongbloed K, Thomas V, Pearce ME, Christian KW, Zhang H, Oviedo-Joekes E, et al. The Cedar Project: Residential transience and HIV vulnerability among young Aboriginal people who use drugs. Health Place. 2015;33:125-31.

42. Connor KM, Davidson JR. Development of a new resilience scale: The Connor-Davidson resilience scale (CD-RISC). Depress Anxiety. 2003;18:76-82.

43. Derogatis LR. SCL-90-R: Symptom Checklist-90-R: administration, scoring, and procedures manual. Minneapolis: NCS Pearson; 1996.

44. Moher D, Schulz KF, Altman DG. The CONSORT statement: revised recommendations for improving the quality of reports of parallel group randomized trials. BMC Med Res Methodol. 2001;1:2.

45. Hewitt CE, Torgerson DJ, Miles JN. Is there another way to take account of noncompliance in randomized controlled trials? Can Med Assoc J. 2006;175:347-348.

46. R Core Development Team. R: A language and environment for statistical computing. Vienna: R Foundation for Statistical Computing; 2012.

47. Canadian Institutes of Health Research, Natural Sciences and Engineering Research Council of Canada Social Sciences and Humanities Research Council of Canada. Chapter 9: Research Involving the First Nations, Inuit and Metis Peoples of Canada. In: Tri-Council Policy Statement on Ethical Conduct for Research Involving Humans; 2010

48. Schnarch B. Ownership, control, access, and possession (OCAP) or selfdetermination applied to research. J Aborig Health. 2004;1:80-95.

49. Lester R, Karanja S. Mobile phones: exceptional tools for HIV/AIDS, health, and crisis management. Lancet Infect Dis. 2008;8:738-9.

50. Lester RT, Gelmon L, Plummer FA. Cell phones: tightening the communication gap in resource-limited antiretroviral programmes? AIDS. 2006;20:2242-4.

51. Jongbloed K, Parmar S, van der Kop M, Spittal PM, Lester R. Recent Evidence for Emerging Digital Technologies to Support Global HIV Engagement in Care. Curr HIV/AIDS Rep. 2015;12:451-61.

52. Mahal D, van der Kop M, Murray M, Jongbloed K, Patel A, Momin Kazi M, et al. Mobile health: An update on BC projects that use WelTel to enhance patient care. BC Med J. 2014,56:90.

53. Smillie K, Borek NV, van der Kop ML, Lukhwaro A, Li N, Karanja S, et al. Mobile health for early retention in HIV care: a qualitative study in Kenya (WelTel Retain). Afr J AIDS Res. 2014;13:331-8.

54. Pop-Eleches C, Thirumurthy H, Habyarimana JP, Zivin JG, Goldstein MP, de Walque $\mathrm{D}$, et al. Mobile phone technologies improve adherence to antiretroviral treatment in a resource-limited setting: a randomized controlled trial of text message reminders. AIDS. 2011;25:825.

55. Mills EJ, Lester R, Ford N. Adherence to antiretroviral therapy: supervision or support? Lancet Infect Dis. 2012;12:97-8.

56. van der Kop ML, Karanja S, Thabane L, Marra C, Chung MH, Gelmon L, et al. In-depth analysis of patient-clinician cell phone communication during the WelTel Kenya1 Antiretroviral Adherence Trial. PLoS One. 2012;7, e46033.

57. Lester RT. Ask, don't tell-mobile phones to improve HIV care. N Engl J Med. 2013;369:1867-8.

58. Chib A, Wilkin H, Ling LX, Hoefman B, Van Biejma H. You have an important message! Evaluating the effectiveness of a text message HIV/AIDS campaign in Northwest Uganda. J Health Commun. 2012;17:146-57.

59. Jamison J, Karlan D, Raffler P. Mixed method evaluation of a passive mHealth sexual information texting service in Uganda. National Bureau of Economic Research Working Paper No. 19107; 2013.

60. Mbuagbaw L, Thabane L, Ongolo-Zogo P, Lester RT, Mills EJ, Smieja M, et al. The Cameroon Mobile Phone SMS (CAMPS) trial: a randomized trial of text messaging versus usual care for adherence to antiretroviral therapy. PLoS One. 2012;7, e46909

61. Torgerson DJ, Roland M. What is Zelen's design? BMJ. 1998;316:606.

62. Hatcher S, Coupe N, Durie M, Elder H, Tapsell R, Wikiriwhi K, et al. Te Ira Tangata: A Zelen randomised controlled trial of a treatment package including problem solving therapy compared to treatment as usual in Maori who present to hospital after self harm. Trials. 2011;12:117.

63. Relton C, Torgerson D, O'Cathain A, Nicholl J. Rethinking pragmatic randomised controlled trials: introducing the "cohort multiple randomised controlled trial" design. BMJ. 2010;340:963-7.

64. Wilson S. Research is ceremony: Indigenous research methods. Winnipeg: Fernwood Publishing; 2008.

\section{Submit your next manuscript to BioMed Central and we will help you at every step:}

- We accept pre-submission inquiries

- Our selector tool helps you to find the most relevant journal

- We provide round the clock customer support

- Convenient online submission

- Thorough peer review

- Inclusion in PubMed and all major indexing services

- Maximum visibility for your research

Submit your manuscript at www.biomedcentral.com/submit 